\title{
IDEAL DECOMPOSITIONS OF KILLING AND HOLOMORPHIC VECTOR FIELDS
}

\author{
WALTER C. LYNGE
}

\section{Introduction}

Let $(M, g)$ be a compact, connected, oriented Riemannian manifold. We show in $\S 3$ that the Lie algebra of Killing vector fields on $M$ can be decomposed into a direct sum of ideals according to the reducibility of the linear holonomy group of $M$. A decomposition of this type is already known for the case of a simply connected, complete Riemannian manifold. If in a addition $M$ is assumed to be Kählerian, we show in $\S 4$ that the Lie algebra of holomorphic vector fields on $M$ can also be decomposed in this way. Our proofs make use of part of a theory of Chern in the form described briefly in $\S 2$.

\section{Preliminaries}

Let $O(M)$ and $\Gamma_{O(M)}$ denote respectively the oriented orthonormal frame bundle over $M$ with structure group $S O(n)$ and the Riemannian connection in $O(M)$. We assume $\Gamma_{O(M)}$ to be reducible to a connection $\Gamma_{P}$ in a subbundle $P$ of $O(M)$ with structure group $G \subset S O(n)$ and projection $\pi$. The case which will interest us most in the sequel is where $P$ is the holonomy bundle through some point of $O(M)$.

Let $\left\{e_{1}, \cdots, e_{n}\right\}$ and $\left\{A_{1}, \cdots, A_{n}\right\}$ be respectively the canonical basis of $R^{n}$ and a basis for the Lie algebra of $G$. Let $\theta=\sum_{i=1}^{n} \theta^{i} e_{i}, \omega=\sum_{\lambda=1}^{m} \omega^{2} A_{\lambda}$, and $\Omega=$ $\sum_{\lambda=1}^{m} \Omega^{\lambda} A_{\lambda}$ be respectively the canonical form of $P$, the connection form of $\Gamma_{P}$, and the curvature form of $\Gamma_{P}$. We have the formula $\Omega^{\lambda}=\frac{1}{2} \sum_{i, j=1}^{n} r_{i j}^{\lambda} \theta^{i} \wedge \theta^{j}$ with $r_{i j}^{\lambda}=-r_{j i}^{\lambda}$. Let $\left(A_{\lambda}\right)_{i j}=a_{j \lambda}^{i}, i, j=1, \cdots, n$. Then there exist functions $s^{\lambda \mu}$ on $P$ such that $r_{k l}^{\lambda}=\sum_{\mu} s^{\lambda \mu} a_{l \mu}^{k}$ with $s^{\lambda \mu}=s^{\mu \lambda}$. Let $A_{\lambda}^{*}$ denote the fundamental vector field on $P$ corresponding to $A_{\lambda}, \lambda=1, \cdots, m$. Let $X_{i}, i=1, \cdots, n$, be vector fields on $P$ such that $\theta^{i}\left(X_{j}\right)=\delta_{j}^{i}, \omega^{\lambda}\left(X_{j}\right)=0$. Define differential operators $P^{*}$ and $S^{*}$ on the space of smooth functions on $P$ by $P^{*}=\sum_{k} X_{k}{ }^{2}$ and $S^{*}=$ $\sum_{\mu, \lambda} s^{\lambda \mu} A_{\mu}^{*} A_{\lambda}^{*}$. These differential operators commute with right translation by

Communicated by Y. Matsushima, December 3, 1971. 
elements of $G$. Thus, if $\rho$ is a representation of $G$ in a vector space $V$, and $f$ is a $V$-valued equivariant function on $P$, then $P^{*} f$ and $S^{*} f$ are also equivariant, where $P^{*} f$ and $S^{*} f$ are defined componentwise for $V$-valued functions after selecting a basis for $V$.

Let $j$ denote the natural representation of $G$ in $R^{n}$. Let $\mathfrak{F}=$ equivariant smooth functions on $P$ of type $\left.\left(j, R^{n}\right)\right\}, \mathscr{D}=$ smooth 1 -forms on $M$ \}, and $\mathfrak{X}$ $=\{$ smooth vector fields on $M\}$. Given $\eta$ in $\mathfrak{D}$, we have $\pi^{*} \eta=\sum_{i=1}^{n} f_{i} \theta^{i}$. To $\eta$ we associate $f=\sum_{i=1}^{n} f_{i} e^{i}$ in $\mathfrak{F}$. This defines a $1: 1$ correspondence between $\mathfrak{D}$ and $\mathfrak{f}$. Moreover, the Riemannian metric gives a 1: 1 correspondence between $\mathfrak{D}$ and $\mathfrak{X}$. Hence we have also a $1: 1$ correspondence between $\mathfrak{X}$ and $\mathfrak{F}$.

Let $\Delta$ and $S$ be respectively the Laplacian on $M$ and the Ricci tensor interpreted as an endomorphism of the cotangent space at each point. By the 1:1 correspondence between $\mathfrak{D}$ and $\mathfrak{F}$, we may regard the maps $\Delta, S: \mathfrak{D} \rightarrow \mathfrak{D}$ as maps $\Delta, S: \mathfrak{F} \rightarrow \mathfrak{F}$. In terms of the operators $P^{*}$ and $S^{*}$, we then have the expressions $\Delta=-P^{*}-S^{*}$ and $S=S^{*}$.

Suppose $c$ is a linear transformation of $R^{n}$ which commutes with the action of $G$. Let $\eta$ be an element of $\widetilde{\mho}$. Define $(c \cdot \eta)(u)=c(\eta(u))$ for each $u$ in $P$. Then $c \cdot \eta$ is in $\mathfrak{F}$, and we have a map $\mathfrak{\mho} \rightarrow \mathfrak{F}$ also denoted by $c$. By a theorem of Chern the diagram

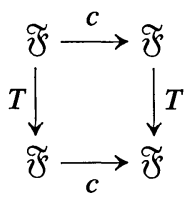

commutes when $T$ is $P^{*}$ or $S^{*}$.

\section{Decomposition of Killing vector fields}

Our decomposition theorem for Killing vector fields is :

Theorem 1. Let $M$ be a compact, connected, oriented Riemannian manifold with oriented orthonormal frame bundle $O(M)$. Let $P$ be any holonomy bundle of $O(M)$ with structure group $\Phi$. Suppose $R^{n}=V_{1} \oplus \cdots \oplus V_{r}$, where the $V_{i}$ are mutually orthogonal subspaces of $R^{n}$ with respect to the usual inner product and each $V_{i}$ is invariant under $\Phi$. Let (S) be the Lie algebra of Killing vector fields on $M$. Then $\mathbb{S}=\mathbb{S}_{1} \oplus \cdots \oplus \mathbb{B}_{r}$, where $\mathbb{B S}_{i}=\{X \mid X \in \mathbb{S}$ and the equivariant $R^{n}$-valued function on $P$ corresponding to $X$ is $V_{i}$-valued $\}$. Moreover, each $\mathbb{B S}_{i}$ is an ideal in $\mathbb{6}$.

Proof. We can find an element $g$ in $S O(n)$ such that $g^{-1} V_{i}=R^{p_{i}}, i=1$, $\cdots, r$, where $R^{p_{i}}$ is the subspace of $R^{n}$ spanned by $\left\{e_{1}, \cdots, e_{p_{1}}\right\}, R^{p_{2}}$ is the subspace spanned by $\left\{e_{p_{1+1}}, \cdots, e_{p_{1}+p_{2}}\right\}$, etc. Let $P^{\prime}$ be the holonomy bundle 
$R_{g} P$ with structure group $g^{-1} \Phi g=\Phi^{\prime}$. Then $\Phi^{\prime}$ leaves each $R^{p_{i}}$ invariant, and $R^{n}=R^{p_{1}} \oplus \cdots \oplus R^{p_{r}}$. Let $\mathbb{S}_{i}^{\prime}=\left\{X \mid X \in \mathbb{B}\right.$ s and the equivariant $R^{n}$-valued function on $P^{\prime}$ corresponding to $X$ is $R^{p_{i}}$-valued $\}$. It is easy to compute that $\mathbb{B S}_{i}=\mathbb{B S}_{i}^{\prime}$. Thus we may consider $V_{i}$ to be $R^{p_{i}}, i=1, \cdots, r$. Furthermore, we restrict ourselves to the case $r=2$ for simplicity and write $R^{p_{1}}=R^{p}, R^{p_{2}}=$ $R^{n-p}$.

Let $X$ be a Killing vector field on $M$ with corresponding 1-form $\alpha$. Let $f$ be the $R^{n}$-valued function on $P$ corresponding to $X$. Let $\rho_{1}, \rho_{2}$ be the projection maps $R^{n} \rightarrow R^{p}$ and $R^{n} \rightarrow R^{n-p}$ respectively. Then $f=f_{1}+f_{2}$, where $f_{i}=\rho_{i} f$, $i=1,2$. Let $X_{1}, X_{2}$ be the vector fields on $M$ corresponding to $f_{1}, f_{2}$ respectively. Then $X=X_{1}+X_{2}$. If we show $X_{1}$ and $X_{2}$ are Killing, it follows easily that $\mathbb{S}=\mathbb{S}_{1} \oplus \mathbb{S}_{2}$. Let $\alpha_{1}, \alpha_{2}$ be the 1 -forms on $M$ corresponding to $X_{1}, X_{2}$ respectively. Applying the Chern theorem, we see that $\Delta \alpha_{i}=2 S \alpha_{i}$, $i=1,2$. To show $X_{1}$ and $X_{2}$ are Killing, it suffices now to prove that $\delta \alpha_{i}=0$, $i=1,2$. In order to do this, we first make a felicitous choice of moving frame:

Let $v$ be an element of $P$ with $\pi(v)=x$. Write $v=\left(Y_{1 x}, \cdots, Y_{n x}\right)$, where $Y_{i x} \in T_{x} M, i=1, \cdots, n$. Let $\left\{x^{1}, \cdots, x^{n}\right\}$ be the normal coordinate system on a neighborhood $U$ of $x$ determined by the frame $v$. Then $\partial /\left.\partial x^{i}\right|_{x}=Y_{i x}$, $i=1, \cdots, n$. Translate $v$ parallelly along geodesics emanating from $x$ to obtain a moving orthonormal frame $\sigma=\left[Y_{1}, \cdots, Y_{n}\right]$ over $U$. By the definition of $P, \sigma$ is a section of $P$ over $U$. It is called the adapted frame on a neighborhood $U$ of $x$ determined by $v$. Define two distributions $V$ and $W$ on $U$ by $V_{y}=$ subspace of $T_{y} M$ spanned by $\left\{Y_{1 y}, \cdots, Y_{p y}\right\}$ and $W_{y}=$ subspace spanned by $\left\{Y_{p+1 y}, \cdots, Y_{n y}\right\}, y \in U$. We have that $T_{y} M=V_{y} \oplus W_{y}$ and $V_{y} \perp W_{y}$ for each $y$ in $U$.

Lemma 1. $V_{y}$ and $W_{y}$ are invariant under $\Phi(y)$, where $\Phi(y)$ is the linear holonomy group of $\Gamma_{O(M)}$ with reference point $y$ regarded as a linear group acting on $T_{y} M$.

Proof. We recall that the action of $\Phi(y)$ on $T_{y} M$ may be described as follows : Let $w=\left(Y_{1 y}, \cdots, Y_{n y}\right)$, and suppose $\tau$ is an element of $\Phi(y)$. Regarding the linear frames $w$ and $\tau(w)$ as maps from $R^{n}$ to $T_{y} M$ in the usual way, we associate to $\tau$ the linear transformation $\tau(w) \circ w^{-1}$ of $T_{y} M$. We need to show that $\tau(w) \circ w^{-1}$ leaves $V_{y}$ and $W_{y}$ invariant. Let $\Phi(w)$ denote the holonomy group of $\Gamma_{O(M)}$ with reference point $w$. Since $w \in P, \Phi(w)=\Phi$. Thus there is an element $g$ in $\Phi$ such that $\tau(w)$ is the linear frame $w \circ g: R^{n} \rightarrow T_{y} M$. But $g$ leaves $R^{p}$ and $R^{n-p}$ invariant. Moreover $w\left(R^{p}\right)=V_{y}$ and $w\left(R^{n-p}\right)=W_{y}$. Hence the result.

Next we need to establish some properties of the Christoffel symbols $\Gamma_{j k}^{i}$ with respect to the frame $\sigma$. We do this in the next four lemmas, omitting most of the details.

Lemma 2. $\Gamma_{i j}^{k}=0$ if $k>p$ and $i, j \leq p$, and $\Gamma_{i j}^{k}=0$ if $k \leq p$ and $i, j>p$, everywhere on $U$.

Proof. Using Lemma 1 we can show that if $X$ and $Z$ are vector fields on 
$U$ belonging to the distribution $V$ (respectively $W$ ), then $\nabla_{Z} X$ and $\nabla_{X} Z$ also belong to $V$ (respectively $W$ ). The result then follows at once.

Lemma 3. $\Gamma_{m i}^{j}=-\Gamma_{m j}^{i} \forall i, m, j$ everywhere on $U$.

Proof. This follows from the fact that $\nabla g=0$ and the fact that the moving frame $\left[Y_{1}, \cdots, Y_{n}\right]$ is orthonormal.

Lemma 4. $\Gamma_{i j}^{k}=\Gamma_{j i}^{k}$ at $x \forall i, j, k$.

Proof. Let $Y=\sum_{i=1}^{n} \mathrm{y}^{i} \partial / \partial x^{i}$ be the vector field on $U$ determined by parallelly translating a vector $Y_{x}$ at $x$ along geodesics emanating from $x$, where again $\left\{x^{1}, \cdots, x^{n}\right\}$ are normal coordinates on $U$ determined by $v$. Using the differential equations for parallel translation, we can show that $\partial y^{k} / \partial x^{l}=0$ at $x \forall k, l$. We then apply this fact to show that $\left[Y_{i}, Y_{j}\right](x)=0 \forall i, j$. Since the torsion is zero, we then have the result.

Lemma 5. $\Gamma_{i m}^{k}=0$ at $x$ unless $k, i, m$ are all $\leq p$ or all $>p$.

Proof. Immediate from Lemmas 2, 3, 4.

We now return to the proof that $\delta \alpha_{i}=0, i=1,2$. Pick an arbitrary point $x$ in M. Let $v$ be an element of $P$ such that $\pi(v)=x$. Let $\left[Y_{1}, \cdots, Y_{n}\right]=\sigma$ be the adapted frame on a neighborhood $U$ of $x$ determined by $v$. Let $\left[\beta_{1}, \cdots, \beta_{n}\right]$ be the moving coframe dual to $\sigma$. It is not difficult to check that we have representations of the form $\alpha_{1}=\sum_{i=1}^{p} \eta_{i} \beta^{i}$ and $\alpha_{2}=\sum_{i=p+1}^{p} \eta_{i} \beta^{i}$. Then $X_{1}$ $=\sum_{i=1}^{p} \eta^{i} Y_{i}$ and $X_{2}=\sum_{i=p+1}^{n} \eta^{i} Y_{i}$, where $\eta^{i}=\eta_{i}, i=1, \cdots, n$, because $\sigma$ is an orthonormal frame. Since $X$ is Killing, we have $\eta_{j ; i}+\eta_{i ; j}=0 \forall i, j$. Setting $i=j$, we have $\eta_{j ; j}=0$. But $\eta_{j ; j}=Y_{j}\left(\eta_{j}\right)-\sum_{i} \eta_{i} \Gamma_{j j}^{i}$. Hence $Y_{j}\left(\eta_{j}\right)=$ $\sum_{i} \eta_{i} \Gamma_{j j}^{i}$. By Lemma 5 we have

$$
\begin{aligned}
& \text { if } j \leq p, \text { then } Y_{j}\left(\eta_{j}\right)(x)=\sum_{i \leq p} \eta_{i}(x) \Gamma_{j j}^{i}(x), \\
& \text { if } j>p \text {, then } Y_{j}\left(\eta_{j}\right)(x)=\sum_{i>p} \eta_{i}(x) \Gamma_{j j}^{i}(x) .
\end{aligned}
$$

We recall that $\eta_{; j}^{j}=Y_{j}\left(\eta^{j}\right)+\sum_{k} \Gamma_{j k}^{j} \eta^{k}$. From this, using (1), (2), Lemmas 3 and 5, and the fact that $\eta^{i}=\eta_{i}$ it can be computed that $\eta_{; j}^{j}(x)=0$ for each $j=1, \cdots, n$. But $\left(\delta \alpha_{1}\right)(x)=-\sum_{j=1}^{p} \eta_{; j}^{j}(x)$ and $\left(\delta \alpha_{2}\right)(x)=-\sum_{j=p+1}^{n} \eta_{; j}^{j}(x)$. Hence $\left(\delta \alpha_{1}\right)(x)=\left(\delta \alpha_{2}\right)(x)=0$ for an arbitrary point $x$ in $M$. Thus $X_{1}$ and $X_{2}$ are Killing.

It remains only to show that $\mathbb{S}_{1}$ and $\mathbb{S}_{2}$ are ideals in $\mathbb{S}^{\prime}$. First we show $\left[\mathbb{S}_{1}, \mathbb{S}_{1}\right] \subset \mathbb{S}_{1}$. Let $X, Z$ be arbitrary elements of $\mathbb{S}_{1}$, and $v$ be an arbitrary point in $P$ with $\pi(v)=x$. Let $\sigma=\left[Y_{1}, \cdots, Y_{n}\right]$ be the adapted frame on a neighborhood $U$ of $x$ determined by $v$. We see easily that $X$ and $Z$ are expressed as 
$X=\sum_{i=1}^{n} \eta^{i} Y_{i}, Z=\sum_{i=1}^{n} \xi^{i} Y_{i}$, where $\eta^{i}=\xi^{i} \equiv 0$ on $U$ for $i>p$. Now $[X, Z]$ $=\sum_{m=1}^{n}[X, Z]^{m} Y_{m}$ where

$$
[X, Z]^{m}=\sum_{i, j}\left(\xi^{i} Y_{i}\left(\eta^{m}\right)+\xi^{i} \eta^{j} \Gamma_{i j}^{m}-\eta^{i} Y_{i}\left(\xi_{m}\right)-\eta^{j} \xi^{i} \Gamma_{j i}^{m}\right)
$$

By Lemma 5 we obtain that $[X, Z]^{m}(x)=0$ for $m>p$. Thus, if $\alpha_{i}, i=1, \cdots, n$, are the components of the 1 -form corresponding to $[X, Z]$, then $\alpha_{i}(x)=0$ for $i>p$. From this it follows that if $f=\sum_{i=1}^{n} f^{i} e_{i}$ is the $R^{n}$-valued function on $P$ corresponding to $[X, Z]$, then $f^{i}(v)=0$ for $i>p$. But $v$ was an arbitrary point of $P$. Hence $[X, Z] \in \mathbb{S S}_{1}$. Similarly we prove that $\left[\mathbb{S}_{2}, \mathbb{G S}_{2}\right] \in \mathbb{S}_{2}$.

To complete the proof of Theorem 1 , we need only to show $\left[\mathbb{S}_{1}, \mathbb{S}_{2}\right]=0$. Let $X, Z$ be arbitrary elements of $\mathbb{S}_{1}, \mathbb{S}_{2}$ respectively. Then $X=\sum_{i=1}^{n} \xi^{i} Y_{i}$ and $Z=\sum_{i=1}^{n} \eta^{i} Y_{i}$ with $\xi^{i} \equiv 0$ on $U$ for $i>p$ and $\eta^{i} \equiv 0$ on $U$ for $i \leq p$, where $\sigma=\left[Y_{1}, \cdots, Y_{n}\right]$ is an adapted frame on a neighborhood $U$ of an arbitrary point $x$ of $M$ determined by some $v$ in $P$ such that $\pi(v)=x$. Again we have $[X, Z]=\sum_{m=1}^{n}[X, Z]^{m} Y_{m}$ where $[X, Z]^{m}$ is given by (3). We will show that $[X, Z]^{m}(x)=0 \forall m$.

Case 1: $m>p$. Then $[X, Z]^{m}(x)=\sum_{i=1}^{p} \xi^{i}(x)\left(Y_{i}\left(\eta^{m}\right)\right)(x)$ by Lemma 4 and the fact that $\xi^{i} \equiv 0$ on $U$ for $i>p$. Since $Z$ is Killing, we have $\eta_{m ; i}+\eta_{i ; m}$ $=0$. Since $\sigma$ is orthonormal, $\eta^{i}=\eta_{i}$. Thus we obtain

$$
Y_{i}\left(\eta^{m}\right)-\sum_{k} \eta_{k} \Gamma_{m i}^{k}+Y_{m}\left(\eta^{i}\right)-\sum_{k} \eta_{k} \Gamma_{i m}^{k}=0
$$

For $m>p$ and $i \leq p$, it follows by Lemma 5 that $Y_{i}\left(\eta^{m}\right)=-Y_{m}\left(\eta^{i}\right)$ at $x$. Thus $[X, Z]^{m}(x)=-\sum_{i \leq p} \xi^{i}(x)\left(Y_{m}\left(\eta^{i}\right)\right)(x)=0$.

Case 2: $m \leq p$. The proof is similar.

\section{Decomposition of holomorphic vector fields}

We now assume our compact, connected, oriented Riemannian manifold $M$ is Kählerian. The complex dimension is $n / 2$, where $n$ is now even. We denote the complex structure by $J$. Let $\mathfrak{A}$ be the real Lie algebra of infinitesimal automorphisms of $M$. $\mathfrak{U}$ is made into a complex Lie algebra using the almost complex structure $J$. Let $\mathfrak{S}$ be the complex Lie algebra of holomorphic vector fields on $M$. As complex Lie algebras, $\mathfrak{S}=\mathfrak{A}$. Thus we will deal with $\mathfrak{U}$ from now on.

The infinitesimal automorphisms of $M$ are characterized by the property that 
their corresponding 1-forms satisfy $\Delta \alpha=2 S \alpha$. Therefore, if $\Gamma_{O(M)}$ is reducible to a connection $\Gamma_{P}$ in a subbundle $P$ of $O(M)$ with structure group $G \subset S O(n)$, and if $R^{n}=V_{1} \oplus \cdots \oplus V_{r}$ where each $V_{i}$ is invariant under the action of $G$, then by the theorem of Chern we have a corresponding decomposition of the real Lie algebra $\mathfrak{X}$ into a direct sum of vector subspaces : $\mathfrak{U}=\mathfrak{U}_{1} \oplus \cdots \oplus \mathfrak{U}_{r}$, where $\mathfrak{A}_{i}=\left\{X \mid X \in \mathfrak{U}\right.$ and the equivariant $R^{n}$-valued function on $P$ corresponding to $X$ is $V_{i}$-valued $\}$.

Now we specialize $P$ and $G$. Let $x$ be an arbitrary point of $M$. Let $T_{x} M=$ $V_{1 x} \oplus \cdots \oplus V_{s x}$ be a direct sum decomposition of $T_{x} M$ such that the $V_{i x}$ are mutually orthogonal subspaces invariant under $\Phi(x)$ and $J_{x}$. There is an element $u$ in $O(M), \pi(u)=x$, which is of the form

$$
u=\left(u_{1}, \cdots, u_{r_{1}}, J u_{1}, \cdots, J u_{r_{1}}, u_{r_{1}+1}, \cdots, u_{r_{1}+r_{2}}, J u_{r_{1}+1}, \cdots, J u_{r_{1}+r_{2}}, \cdots\right)
$$

where the first $2 r_{1}$ vectors in the frame $u$ span $V_{1 x}$, the next $2 r_{2}$ vectors span $V_{2 x}$, etc.

Theorem 2. Let $P=P(u)$ be the holonomy bundle of $O(M)$ through $u$ with structure group $G=\Phi(u)=\Phi$. Let $R^{n}=R^{2 r_{1}} \oplus \cdots \oplus R^{2 r_{s}}$. Then each $R^{2 r_{i}}, i=1, \cdots, s$, is invariant under $\Phi$. Moreover, if $\mathfrak{A}=\mathfrak{A}_{1} \oplus \cdots \oplus \mathfrak{A}_{s}$ is the decomposition of $\mathfrak{A}$ corresponding to this decomposition of $R^{n}$, then the $\mathfrak{U}_{i}$ are complex subspaces of $\mathfrak{A}$ and in fact ideals.

Proof. For simplicity we assume $s=2$ and write $R^{2 r_{1}}=R^{p}, R^{2 r_{2}}=R^{n-p}$, $V_{1 x}=V_{x}$, and $V_{2 x}=W_{x}$. To show $R^{p}$ is invariant under $\Phi$, let $g$ be an arbitrary element of $\Phi$. Then there is an element $\tau$ of $\Phi(x)$ such that $\tau(u)$ is the frame $u \circ g: R^{n} \rightarrow T_{x} M$. Since $V_{x}$ is invariant under $\Phi(x)$, we have $\tau(u) \circ u^{-1} V_{x}=u \circ g \circ u^{-1} V_{x}=V_{x}$. But $u^{-1} V_{x}=R^{p}$. Therefore $u \circ g R^{p}=V_{x}$ and $g R^{p}=u^{-1} V_{x}=R^{p}$. Similarly $R^{n-p}$ is invariant under $\Phi$.

Now let $v$ be an arbitrary element of $P$ with $\pi(v)=y$. By the definition of $P=P(u)$ and the fact that $\nabla J=0$ we see that $v$ is of the form

$$
v=\left(v_{1}, \cdots, v_{r_{1}}, J v_{1}, \cdots, J v_{r_{1}}, v_{r_{1}+1}, \cdots, v_{r_{1}+r_{2}}, J v_{r_{1}+1}, \cdots, J v_{r_{1}+r_{2}}\right)
$$

where $2 r_{1}=p$ and $2 r_{2}=n-p$. Thus, if $V_{y}$ is the subspace of $T_{y} M$ spanned by the first $p$ vectors of this frame and $W_{y}$ is the subspace spanned by the last $n-p$ vectors, then we have $J_{y} V_{y}=V_{y}$ and $J_{y} W_{y}=W_{y}$. It follows readily that $J \mathfrak{A}_{i} \subset \mathfrak{A}_{i}, i=1,2$. Thus $\mathfrak{A}_{1}$ and $\mathfrak{U}_{2}$ are complex subspaces of $\mathfrak{A}$.

It remains to show that $\mathfrak{A}_{1}$ and $\mathfrak{U}_{2}$ are ideals in $\mathfrak{A}$. The proof that $\left[\mathfrak{U}_{i}, \mathfrak{A}_{i}\right]$ $\subset \mathfrak{U}_{i}, i=1,2$, is exactly the same as the proof of the analogous fact in Theorem 1 . We therefore have only to show $\left[\mathfrak{U}_{1}, \mathfrak{A}_{2}\right]=0$. Let $X, Z$ be arbitrary elements of $\mathfrak{U}_{1}, \mathfrak{U}_{2}$ respectively. Let $y$ be an arbitrary point of $M$ and let $\sigma=$ $\left[Y_{1}, \cdots, Y_{n}\right]$ be an adapted frame on a neighborhood $U$ of $y$ determined by an element $v=\left(Y_{1 y}, \cdots, Y_{n y}\right)$ in $P$. Let the components of $X, Z$, and $[X, Z]$ be $\xi^{i}, \eta^{i}$ and $[X, Z]^{m}$ respectively in the frame $\sigma$. Then $\xi^{i} \equiv 0$ for $i>p$ and $\eta^{i} \equiv 0$ for $i \leq p$. From Lemma 4 we obtain 


$$
[X, Z]^{m}(y)=\sum_{i=1}^{n} \xi^{i}(y)\left(Y_{i}\left(\eta^{m}\right)\right)(y)-\eta^{i}(y)\left(Y_{i}\left(\xi^{m}\right)\right)(y) .
$$

Thus $[X, Z]^{m}(y)=-\sum_{i>p} \eta^{i}(y)\left(Y_{i}\left(\xi^{m}\right)\right)(y)$ for $m \leq p$, and $[X, Z]^{m}(y)=$ $\sum_{i \leq p} \xi^{i}(y)\left(Y_{i}\left(\eta^{m}\right)\right)(y)$ for $m>p$. In order to show $[X, Z]^{m}(y)=0 \forall m$, it suffices to show

$$
\begin{aligned}
& \left(Y_{i}\left(\xi^{m}\right)\right)(y)=0 \text { for } i>p \text { and } m \leq p \text {, and } \\
& \left(Y_{i}\left(\eta^{m}\right)\right)(y)=0 \text { for } i \leq p \text { and } m>p \text {. }
\end{aligned}
$$

Lemma 6. $\Phi^{0}(y)$, the restricted linear holonomy group at $y$ (which is the identity component of $\Phi(y)$ ) with respect to $\Gamma_{o(M)}$, is decomposed into the direct product of two normal subgroups $\Phi_{1}^{0}(y)$ and $\Phi_{2}^{0}(y)$ such that $\Phi_{1}^{0}(y)$ is trivial on $W_{y}$ and $\Phi_{2}^{0}(y)$ is trivial on $V_{y}$.

Proof. This follows from the proof of Proposition 5.3, p. 183 in [2]. By Lemma 1 we have that $\Phi_{1}^{0}(y) V_{y} \subset V_{y}$ and $\Phi_{2}^{0}(y) W_{y} \subset W_{y}$. From this and Lemma 6 it follows that the holonomy algebra $\phi(y)$ at $y$ splits into a direct sum of subalgebras $\phi_{1}(y)$ and $\phi_{2}(y)$, where, as linear endomorphisms of $T_{y} M$ with respect to the basis $\left\{Y_{1 y}, \cdots, Y_{n y}\right\}$, elements of $\phi_{1}(y)$ (respectively $\phi_{2}(y)$ ) are represented by matrices of the form

$$
\left\|\begin{array}{ll}
a_{p \times p} & 0 \\
0 & 0
\end{array}\right\| \quad\left(\text { respectively }\left\|\begin{array}{ll}
0 & 0 \\
0 & b_{(n-p) \times(n-p)}
\end{array}\right\|\right) .
$$

Now let $\eta$ be the 1-form corresponding to the infinitesimal automorphism $Z$. By an easy computation we find that with respect to the moving frame $\sigma,(\nabla \eta)_{l i}$ $=Y_{i}\left(\eta^{l}\right)+\sum_{j} \eta^{j} \Gamma_{i j}^{l}$. In particular, $(\nabla \eta)_{l i}(y)=\left(Y_{i}\left(\eta^{l}\right)\right)(y)$ if $l>p$ and $i \leq p$. But an element of $\phi(y)+J_{y} \phi(y)$ is represented with respect to the basis $\left\{Y_{1 y}, \cdots, Y_{n y}\right\}$ of $T_{y} M$ by a matrix of the form

$$
\left\|\begin{array}{ll}
A_{p \times p} & O_{p \times(n-p)} \\
O_{(n-p) \times p} & B_{(n-p) \times(n-p)}
\end{array}\right\| .
$$

This follows from the above representations of $\phi_{i}(y), i=1,2$, and from the fact that $J_{y} V_{y}=V_{y}$ and $J_{y} W_{y}=W_{y}$. By a theorem of Lichnerowicz [3, p. 151], $(\nabla \eta)_{y} \in \phi(y)+J_{y} \phi(y)$. This implies that $(\nabla \eta)_{l i}(y)=0$ if $l>p$ and $i \leq p$. Thus we must have $\left(Y_{i}\left(\eta^{m}\right)\right)(y)=0$ for $i \leq p$ and $m>p$. Similarly, we can prove that $\left(Y_{i}\left(\xi^{m}\right)\right)(y)=0$ for $i>p$ and $m \leq p$. This completes the proof of Theorem 2.

Finally, if we assume the Kähler manifold $M$ is nondegenerate, we can obtain a decomposition of $\mathfrak{U}$ starting, as in the Killing case, from any holonomy bundle: 
Theorem 3. Let $M$ be nondegenerate. Suppose $P$ is any holonomy bundle of $O(M)$, and $G=\Phi$ is its structure group. Suppose $R^{n}=V_{1} \oplus \cdots \oplus V_{r}$ is a decomposition of $R^{n}$ into a direct sum of subspaces mutually orthogonal with respect to the usual inner product and invariant under $\Phi$. Let $\mathfrak{U}=\mathfrak{U}_{1} \oplus \cdots \oplus \mathfrak{A}_{r}$ be the corresponding decomposition of $\mathfrak{U}$. Then the $\mathfrak{A}_{i}$ are complex subspaces of $\mathfrak{A}$ and in fact ideals.

Proof. As in Theorem 1, it suffices to consider $V_{1} \oplus \cdots \oplus V_{r}$ to be $R^{p_{1}} \oplus \cdots \oplus R^{p_{r}}$. For simplicity, we consider only the case $r=2$ and write $R^{n}=R^{p} \oplus R^{n-p}$. Let $u=\left(Y_{1 x}, \cdots, Y_{n x}\right)$ be an element of $P$ with $\pi(u)=x$. Then by Lemma $1, V_{x}$ and $W_{x}$, the subspaces of $T_{x} M$ spanned by $\left\{Y_{1 x}, \cdots, Y_{p x}\right\}$ and $\left\{Y_{p+1 x}, \cdots, Y_{n x}\right\}$ respectively, are invariant under $\Phi(x)$. Since $M$ is nondegenerate, we have $J_{x} \in \Phi(x)\left[3, \mathrm{p}\right.$. 173]. Then, in particular, $J V_{x} \subset V_{x}$ and $J W_{x} \subset W_{x}$. The rest of the proof proceeds as the proof of Theorem 2.

The results of this paper constitute part of the author's Ph. D. dissertation written at the University of Notre Dame. The author would like to express his gratitude to Professor Yozo Matsushima for much encouragement and many helpful suggestions.

\section{Bibliography}

[1] S.S. Chern, On a generalization of Kähler geometry, Algebraic geometry and Topology, a Symposium in Honor of S. Lefschetz, Princeton University Press, Princeton, 1957, 103-121.

[2] S. Kobayashi \& K. Nomizu, Foundations of differential geometry, Vol. I, Interscience, New York, 1963.

[ 3 ] - , Foundations of differential geometry, Vol. II, Interscience, New York, 1969.

[4] A. Lichnerowicz, Géométrie des groupes de transformations, Dunod, Paris, 1958.

[ 5 ] A. Weil, Un théorème fondamental de Chern en géométrie Riemannienne, Séminaire Bourbaki, 14ième année, 1961-62, Exp. 239. 